\section{Hellwach trotz} Antihistaminikum?

Dimetindenmaleat (DMM), ein Antihistaminikum der 1. Generation, steht seit kurzem in neuer Galenik als Mikropellets zur Verfügung. Vorteil der neuen Darreichungsform: Das Medikament macht nicht mehr müde, betonte F. Horak, HNO-Universitätsklinik Wien. Unbeeinflußt sind die in zahlreichen Studien belegte Sicherheit und antiallergische Wirksamkeit von DMM bei Patienten mit allergischer Rhinitis und Urtikaria.

Bei den 4 mg-Mikropellets handelt es sich um Retardkapseln, die therapeutische Plasmakonzentrationen schonend aufbauen und so sedierende Effekte auf Plazebo-Niveau senken, erläuterte Horak. In einer eigenen Untersuchung in der Wiener Provokationskammer bei Patienten mit Gräserpollinose waren die $\mathrm{Mi}$ kropellets der Plazebo-Gabe hinsichtlich der Symptomlinderung statistisch signifikant überlegen. Horak bestätigte den DMM-Mikropellets eine Wirkdauer von mindestens 16 Stunden, so daß die Einmalgabe pro Tag ausreicht. Er verwies auf eine Feldstudie von Schöpf et al., die die Wiener Ergebnisse bei 24 Patienten mit Gräserpollen- bzw. Baumpollenallergie bestätigte. In dieser Studie erfolgte die Allergenprovokation eine Stunde nach Medikation. Die Ergebnisse weisen, so Horak, auf einen protektiven Effekt gegenüber nasalen Symptomen bei allergischer Rhinitis hin.
Horak sieht unter der neuen Galenik von DMM keine Gefahr für die Verkehrstüchtigkeit bzw. die Alltagssicherheit. DMMMikropellets könnten nach seiner Ansicht auch Berufstätigen mit allergischer Rhinokonjunktivitis gegeben werden.

(BP)

Quelle: Vortrag F. Horak, Wien, Rheinische Gespräche zur Allergologie und Umweltmedizin, 21. 3.1998. Leitung: Prof. R. Mösges, Köln; Dr. L. Klimek, Mainz (Die Veran staltung wurde durch unterschiedlich Sponsoren aus der Industrie unterstützt).

\section{Innovativer}

\section{Ansatz gegen Asthma}

Nur wenige chronische Erkrankungen verlangen vom Patienten das Erlernen und Einüben ähnlich komplizierter Applikationstechniken wie dies beim Asthma bronchiale mit der Inhalationstherapie der Fall ist. Ein Wirkprinzip, das mit einer einfachen oralen Gabe auskommt, ist hier als Erleichterung $\mathrm{zu}$ werten. Mit Montelukast steht seit kurzem ein Medikament zur Verfügung, das nur einmal täglich (abends) eingenommen werden muß.

Der Wirkstoff ist der erste Vertreter der neuen Substanzklasse der Leukotrien-Antagonisten. Die Leukotriene gelten als stärkste heute bekannte Bronchokonstriktoren. Die von Mastzellen und Eosinophilen freigesetzten Mediatoren fördern ferner Ödembildung, Hypersekretion und Dyskrinie und wirken chemotaktisch für Eosinophile. Deshalb suchte man schon länger nach Wegen, ihre Effekte zu unterbinden. Von dem spezifi- schen Antagonisten Montelukast reichen $10 \mathrm{mg}$ (1 Filmtablette) pro Tag aus, um die Rezeptoren nahezu komplett für 24 Stunden zu blockieren; bei Kindern genügt die halbe Tagesdosis, die in speziellen Kautabletten angeboten wird. Für die Praxis günstig ist der rasche Wirkeintritt.

Sicherheit und Wirksamkeit hat Montelukast (Singulair $^{\circledR}$ ) in diversen Studien unter Beweis gestellt. Der Verbrauch an Betamimetika und der SymptomenScore inkl. nächtlicher Atemnot gingen signifikant zurück; Lungenfunktion sowie die mit standardisiertem Fragebogen bestimmte Lebensqualität besserten sich merklich. Dabei erwies sich die Substanz als ausgesprochen verträglich, wie dies wegen ihres spezifischen Ansatzes zu erwarten war. Die Kombination 10 mg Montelukast plus 400 $\mu \mathrm{g}$ Beclometason zeigte sich der Monotherapie mit dem Steroid überlegen.

Den Stellenwert der Neueinführung sehen die Experten zunächst in der Add-on-Therapie des leichten bis mäßiggradigen Asthmas, d.h. als Ergänzung zur Basistherapie mit inhalierbarem Kortikoid, wenn damit und mit bedarfsmäßiger Inhalation eines Betamimetikums die Symptome nicht befriedigend kontrolliert werden. Besonderen Nutzen verspricht das Mittel auch für die Behandlung des Anstrengungs-Asthmas, wo es sich im klinischen Prüfprogramm bereits als Prophylaktikum bewährt hat.

(wpa)

Pressekonferenz „Singulair ${ }^{\circledR}$ - Der neue Baustein in der Therapie des Asthma bronchiale , Freiburg, 3.4.1998 (Veranstalter: MSD Sharp \& Dohme, Haar bei Mü chen).

\section{Neuer H1- Blocker auf dem Markt}

Seit dem 5. Mai ist Mizolastin, ein neues Antihistaminikum, unter dem Handelsnamen Zolim ${ }^{\circledR}$ neu auf dem Markt erhältlich. Neben einer hohen Affinität zu H1-Rezeptoren soll sich das neue Präparat durch die Fähigkeit auszeichnen, die Bildung und Ausschüttung von Entzündungsmediatoren wie z.B. Leukotrienen enzymatisch $\mathrm{zu}$ blockieren. Zusätzlich kommt es zu einer Stabilisierung der Mastzellen. Insgesamt führt dies sowohl zu einer Hemmung der schnellen, als auch der langsamen allergischen Reaktion.

Nach Informationen der Schwarz Pharma Deutschland GmbH, Monheim.

\section{Brausetablette im 30er Pack}

Ab 1. Juni 1998 bietet die Essex Pharma GmbH die Brausetabletten Lisino Brause $^{\circledR}$ in der Packungsgröße von 30 Tabletten an. Diese Applikationsform soll sich durch einen besonders schnellen Wirkeintritt innerhalb von 10 bis $15 \mathrm{Mi}$ nuten auszeichnen.

Die Brausetabletten sind besonders zum Einsatz bei Heuschnupfen, in der Prophylaxe von Lichtdermatosen bei längerer UV-Exposition und zur Behandlung leichter Formen von Nahrungsmittelallergien geeignet.

Nach Informationen der Essex-Pharma GmbH, München. 